\title{
Future Plans at BNL: RHIC-II and eRHIC
}

\author{
Samuel Aronson \\ Brookhaven National Laboratory \\ Upton, New York, USA
}

The development of future facilities relevant to the study of deep inelastic scattering at BNL is described.

\section{Introduction}

A program of high-energy nuclear physics has been carried out at BNL's Relativistic Heavy Ion Collider (RHIC) since 2000 with relativistic heavy ions and polarized protons. Figure 1 shows an aerial view of the RHIC facility; Table 1 compares the design performance parameters, the performance parameters achieved to date (which are beyond design performance in most cases) and so-called "enhanced performance", based on on-going incremental improvements to the current performance.

The scientific output of the RHIC facility and its four experiments has been outstanding. Strong evidence has been found in the final state of ultra-relativistic heavy-ion collisions of a new state of nuclear matter - a strongly coupled Quark-Gluon Plasma [2] - characterized by strong suppression of jets and particle flow in the final state. The measured properties are consistent with these characteristics arising from a partonic state characterized by very high energy density and extremely low viscosity. This striking and unexpected finding has been labeled the "Perfect Liquid" $[2,3]$.

In the polarized proton program at RHIC, similarly important findings are emerging. In particular, RHIC can provide collisions of longitudinally polarized protons at center of mass

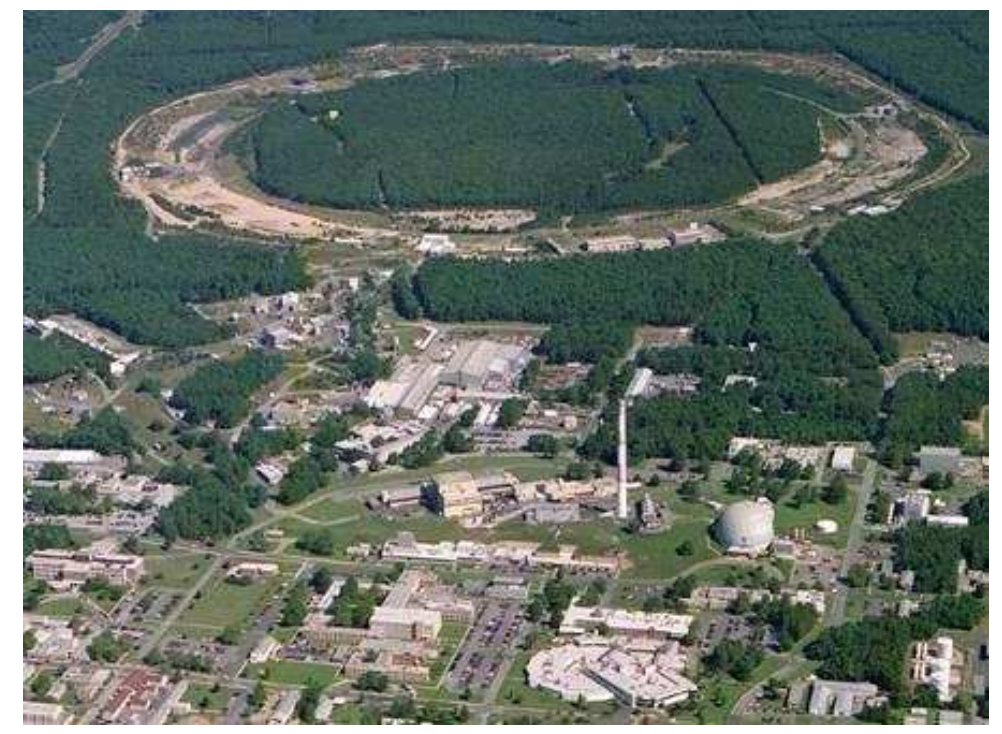

Figure 1: Aerial view of RHIC. 


\begin{tabular}{|c|c|c|c|c|c|c|c|}
\hline Mode & $\begin{array}{l}\text { No. of } \\
\text { bunches }\end{array}$ & $\frac{\text { lons }}{\text { bunch }}$ & $\beta^{*}[\mathrm{~m}]$ & Beam pol. & $\begin{array}{c}L_{\text {store }}^{\text {ave }} \\
{\left[\mathrm{cm}^{-2} \mathrm{~s}^{-1}\right]}\end{array}$ & $\begin{array}{c}A_{1} A_{2} L_{\text {store }}^{\text {ave }} \\
{\left[\mathrm{cm}^{-2} \mathrm{~s}^{-1}\right]}\end{array}$ & $\begin{array}{c}A_{1} A_{2} L_{\text {peak }}^{\text {ave }} \\
{\left[\mathrm{cm}^{-2} \mathrm{~s}^{-1}\right]}\end{array}$ \\
\hline \multicolumn{8}{|c|}{ Design values (1999) } \\
\hline $\mathrm{Au}-\mathrm{Au}$ & 56 & 1.0 & 2 & & $2 \times 10^{26}$ & $8 \times 10^{30}$ & $31 \times 10^{30}$ \\
\hline $\mathrm{p}-\mathrm{p}$ & 56 & 100 & 2 & & $4 \times 10^{30}$ & $4 \times 10^{30}$ & $5 \times 10^{30}$ \\
\hline \multicolumn{8}{|c|}{ Achieved values } \\
\hline $\mathrm{Au}-\mathrm{Au}$ & 103 & $\overline{1.1}$ & 0.8 & & $12 \times 10^{26}$ & $46 \times 10^{30}$ & $120 \times 10^{30}$ \\
\hline $\mathrm{p} \uparrow-\mathrm{p} \uparrow$ & 111 & 130 & 1 & $65 \%$ & $20 \times 10^{30}$ & $20 \times 10^{30}$ & $35 \times 10^{30}$ \\
\hline \multicolumn{8}{|c|}{ Enhanced design values (2009) } \\
\hline $\mathrm{Au}-\mathrm{Au}$ & 111 & 1.1 & 0.9 & & $8 \times 10^{26}$ & $31 \times 10^{30}$ & $155 \times 10^{30}$ \\
\hline $\mathrm{p} \uparrow-\mathrm{p} \uparrow$ & 111 & 200 & 0.9 & $70 \%$ & $60 \times 10^{30}$ & $60 \times 10^{30}$ & $90 \times 10^{30}$ \\
\hline
\end{tabular}

Table 1: RHIC design values, achieved values, and "enhanced" design values.

energies up to $500 \mathrm{GeV}$. Initial results on the double spin asymmetry, which is sensitive to the polarization of gluons in the proton wave function, are consistent with so-called "standard" predictions. If this result is upheld, it means that gluon polarization is not the explanation of the proton "spin crisis" [4].

These and other results, summarized elsewhere in these proceedings, point to the need for further measurements to elucidate these manifestations of QCD both at high and low temperature and density. The fundamental QCD questions now on the table are:

- How does ordinary matter get its mass, spin and other intrinsic and dynamical properties?

- How does QCD matter behave at the extraordinary temperatures attained during the first microseconds following the Big Bang?

- What is the structure of the QCD vacuum, and how is it affected by high temperature and density?

- What are the universal properties of all strongly interacting systems in the limit of high gluon density?

A key component of BNL's future plans in nuclear physics is to provide the means to extend these research programs to address these fundamental questions. The four components of this plan are as follows:

- Near-term upgrades to RHIC: Detector upgrades, Electron Beam Ion Source (EBIS, a new ion source), enhanced luminosity and polarization

- RHIC-II: Beam cooling to maintain small hadron beam emittance at top energy

- eRHIC: An electron ion collider (EIC) capable of producing e-A and polarized e-p collisions at high luminosity, plus new detectors

- Theory and computational QCD

The components of this plan are briefly described in the following sections. 


\section{Near-term RHIC Program}

Table 2 summarizes the current planning assumptions for RHIC operations in the next three fiscal years.

The planning basis for these runs is the "enhanced performance" of RHIC shown in Table 1 above for the accelerator. The EBIS project [5] is presently under construction and will be operational in 2009/2010. At present, the experimental program is also embarked on a series of detector upgrades, based on the results to date and on the need to exploit the highest luminosities that can be made available at RHIC [6]. Several of these upgrades are currently in operation or under construction. A number of questions

\begin{tabular}{|c|c|c|}
\hline Run & Species & $\begin{array}{c}\text { Energy } \\
\sqrt{s_{N N}}(\mathrm{GeV})\end{array}$ \\
\hline 2008 & $\mathrm{~d}+\mathrm{Au}$ & 200 \\
& $\mathrm{p} \uparrow+\mathrm{p} \uparrow$ & 200 \\
\hline 2009 & $\mathrm{Au}+\mathrm{Au}$ & $\mathrm{TBD}$ \\
& $\mathrm{p} \uparrow+\mathrm{p} \uparrow$ & $\mathrm{TBD}$ \\
\hline 2010 & heavy ion & TBD \\
& $\mathrm{p} \uparrow+\mathrm{p} \uparrow$ & 500 \\
\hline
\end{tabular}

Table 2: RHIC operations in the next three fiscal years. raised by the results to date can be addressed by the experimental program between now and 2010 [7].

\section{RHIC-II}

A number of the most crucial questions about the structure of strongly interacting matter above the critical temperature, and about the wave function of the proton, require performance above "enhanced" levels. Below is a somewhat more specific formulation of the RHIC-relevant fundamental questions enumerated above:

- What is the mechanism of the unexpectedly fast thermal equilibration?

- What is the initial temperature and thermal evolution of the medium?

- What is the equation of state, its viscosity and other transport coefficients?

- Is there direct evidence for deconfinement? What is the screening length?

- Is chiral symmetry restored, as predicted by QCD?

- How does the new form of matter hadronize at the phase transition?

- Where is the QCD critical point?

- What is the initial state in heavy ion collisions?

- How does the nucleon get its spin?

The BNL plan, during the Near-term period described above is to develop the technology for increasing the luminosity of RHIC by a factor of 10 beyond the enhanced performance for heavy ion operation and a factor of about 3 for polarized proton operations. The detector upgrades under way during this period will enable the experiments to exploit this higher luminosity.

The luminosity-enhanced RHIC program, dubbed "RHIC-II" requires cooling of the RHIC beams at full energy, via electron-cooling [8] and other techniques. The R\&D for this technology is presently under way. Figure 2 shows a schematic layout of RHIC-II. 
Strong support for construction of RHIC-II is expected in the U.S. Nuclear Science Long Range Plan process. This process is under way, with a report expected to be issued during 2007 .

\section{4 eRHIC}

The outstanding successes of the HERA program [9], coupled with the results from RHIC comprise major advances in understanding QCD. Moreover, it is possible to extend these accomplishments to address central questions in the role of gluons in the structure and interaction of strongly interacting matter:

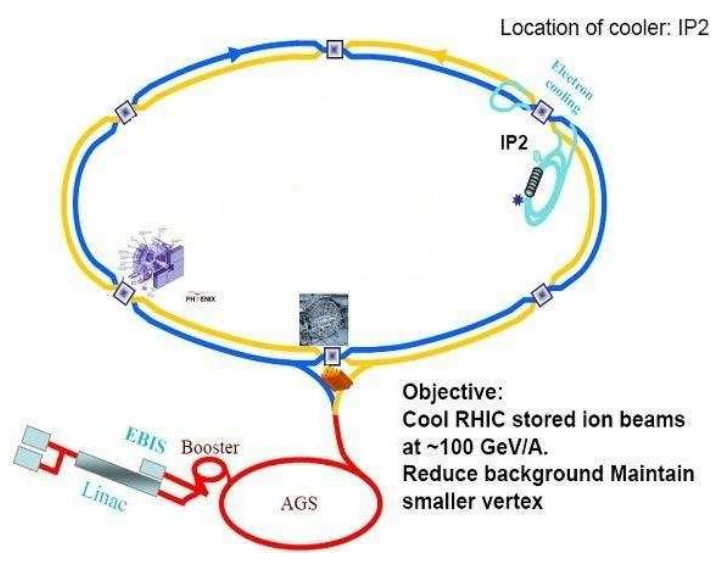

Figure 2: Schematic layout of RHIC-II.

- Does the self-limiting growth of color field strengths in QCD lead to universal behavior of all nuclear and hadronic matter in the vicinity of these limits?

- How does the nuclear environment affect the distribution of gluons in momentum and space?

- What is the internal landscape of a nucleon in the region dominated by sea quarks and gluons?

- How do hadronic final states form from light quarks and massless gluons in QCD?

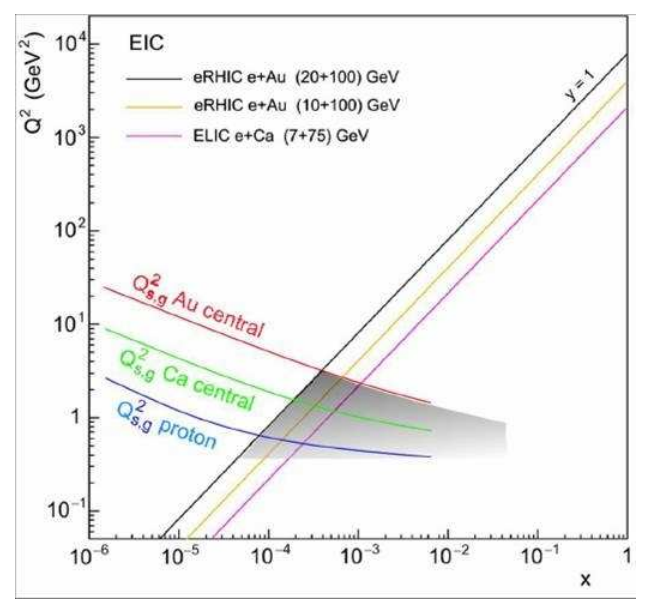

Figure 3: Kinematic reach of eRHIC and ELIC.
The physics case is further elaborated in white papers prepared for the on-going NP Long Range Plan process [10]. To address these questions requires an extension of the HERA program in the form of a new electron-ion collider (EIC) capable of exploring the $\mathrm{x}-\mathrm{Q}^{2}$ region relevant to gluon saturation $\left(\mathrm{x}<10^{-3}, \mathrm{Q}^{2}>1 \mathrm{GeV}^{2}\right)$ with a factor 100 or so greater integrated luminosity than HERA provided in its time.

Brookhaven's plan to address this needed capability is a proposal to build eRHIC, an EIC based on the existing RHIC facility [11]. Other EIC capabilities are also being discussed at Thomas Jefferson National Laboratory (ELIC) [12] and at CERN (LHeC) [13]. Figure 3 shows the kinematic reach of eRHIC and ELIC. 
The eRHIC conceptual design is fairly well-advanced and uses realistic, RHIC-based performance parameters. To achieve the desired kinematic reach, eRHIC would collide polarized electrons up to $20 \mathrm{GeV}$ with either polarized protons at beam energies of 50 $250 \mathrm{GeV}$ and polarization $\geq 70 \%$, or heavy ions at beam energies up to $100 \mathrm{GeV}$ per nucleon. (In addition, EBIS would also allow high energy collisions of electrons with polarized ${ }^{3} \mathrm{He}$ at beam energies up to $167 \mathrm{GeV}$ per nucleon.) To achieve the desired integrated luminosity in a reasonable period of time, eRHIC would provide average luminosity $\geq 10^{33} / \mathrm{cm}^{2} \cdot \mathrm{sec}$.

BNL envisions e-A and polarized e$\mathrm{p}$ collisions at one or more intersection regions at RHIC with a high energy electron synchrotron or an energy ecovery linac (ERL). The ERL solution is currently the favored option and a schematic layout of this option is shown in Figure 4. New detectors optimized for the physics goals would be built at the intersection region or regions providing collisions with electrons. Meanwhile, the RHIC heavy ion and polarized p-p programs could continue at the existing PHENIX and STAR detectors.

An EIC is the highest priority of

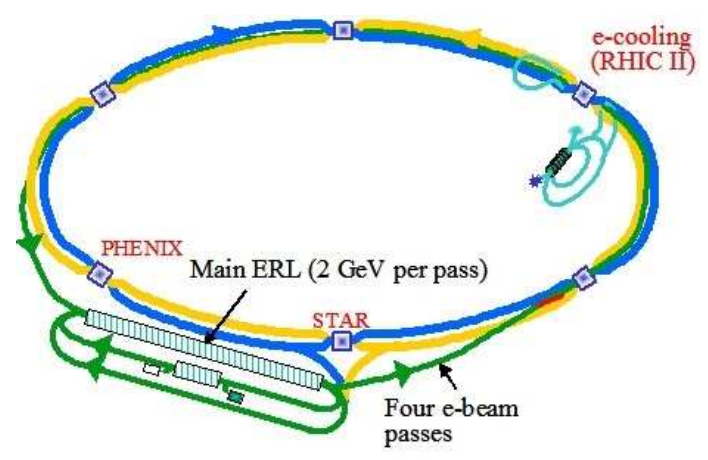

Figure 4: Schematic layout of the linac-ring option for eRHIC.

the U.S. QCD community for new construction, after the completion of RHIC-II and the CEBAF energy upgrade. EIC capabilities are being considered by the broader U.S. Nuclear Physics community in the context of the NP Long Range Plan. It is expected that the Long Range Plan report will strongly endorse accelerator and detector $\mathrm{R} \& \mathrm{D}$ in the immediate future, with a commitment to construct such a facility to be considered in the future, probably during the next long range planning cycle (i.e., in 2012).

\section{Nuclear Theory and Computational QCD}

The nuclear theory effort at BNL has been a vital component of the progress made in understanding and interpreting the results coming from RHIC. In recent years the outstanding strengths of the theory group in strong interaction theory and phenomenology have been complemented by a new effort in computational lattice QCD. This has been made very effective by the presence at BNL of two "QCDOC" supercomputers [14] built by Columbia University, IBM and BNL and operated by the DOE and the RIKEN-BNL Research Center (RBRC) at BNL. These machines, each with a 20 Teraflops peak performance, are focused on lattice $\mathrm{QCD}$, with the RBRC-operated machine largely devoted to finite temperature QCD. They are enabling an accelerated and more realistic study of the physics done at RHIC than has been possible to date.

BNL and Stony Brook University (SBU) have just acquired a New York State-funded 100 Teraflops BlueGene/L computer. This will be operated as the core of a center for computational science. Within a more general research program, it will provide even more capability for lattice QCD research at Brookhaven. Plans are being made for future growth of the computational capabilities available at the SBU-BNL center. 


\section{Summary}

A key goal of BNL's plans for future research and future user facilities in nuclear science is to maintain and extend our world-class capability in the study of QCD. This is based on a proposed evolution of the highly successful RHIC program into a broader QCD laboratory, comprising high energy studies of QCD through collisions involving heavy ions, polarized or unpolarized protons and polarized electrons. The principal components of this evolution are a luminosity upgrade (RHIC-II) for nucleus-nucleus and nucleon-nucleon collisions plus an electron capability (eRHIC) for deep inelastic lepton-nucleus and lepton-nucleon collisions. These, in turn, require detector upgrades, new detectors and an increasing computational capability for lattice QCD.

\section{Acknowledgements}

This work was supported by the Division of Nuclear Physics of the Office of Science of the U.S. Department of Energy under contract DE-AC02-98-CH10886.

\section{References}

[1] Slides: http://indico. cern. ch/contributionDisplay. py? contribId=270\&sessionId=10\&conf Id=9499

[2] I. Arsene et al. [BRAHMS Collaboration], Nucl. Phys. A 7571 (2005).

B. B. Back et al. [PHOBOS Collaboration], Nucl. Phys. A 75728 (2005).

J. Adams et al., [STAR Collaboration], Nucl Phys. A 757102 (2005).

K. Adcox et al., [PHENIX Collaboration], Nucl. Phys. A 757184 (2005).

[3] E. Shuryak, Prog. Part. Nucl. Phys. 53272 (2004).

[4] E. Leader and M. Anselmino, Z. Phys. C 41239 (1988). B.I. Abelev et al., [STAR Collaboration], Phys. Rev. Lett. 97252001 (2006).

[5] M. Wilinski, J. Alessi, E. Beebe, S. Bellavia and A. Pikin, Electron Beam Ion Source Pre-Injector Diagnostics, Beam Instrumentation Workshop 2006, Batavia, Illinois (2006).

[6] Mid-term plan for RHIC: http://www.bnl.gov/HENP/docs/RHICplanning/RHIC_Mid-termplan_print.pdf

[7] Future Science at the Relativistic Heavy Ion Collider: http://www.bnl.gov/physics/rhicIIscience/

[8] See abstracts from Particle Accelerator Conference 07, Albuquerque, New Mexico: 25 presentations on BNL's progress in implementing electron cooling.

[9] A. Deshpande, R. Milner, R. Venugopalan, and W. Vogelsang, Ann. Rev. Nucl. and Part. Sci. 55165 (2005).

[10] P. Jacobs et al., Phases of QCD, Summary of the Rutgers Long Range Plan Town Meeting, New Brunswick, New Jersey (2007).

X. Ji et al., Whitepaper from DNP Town Meeting on Hadronic Physics, New Brunswick, New Jersey (2007).

Both can be found at http://dnp.nscl.msu.edu/

[11] J. Beebe-Wang et al., The eRHIC machine Accelerator Position Paper: http://www.phenix.bnl.gov/WWW/publish/abhay/Home_of_EIC/eic-info.html

[12] ELIC: http://casa.jlab.org/research/elic/elic.shtml

[13] LHeC: http://www-zeuthen.desy.de/lhec/LHeCprop.pdf

[14] P. A. Boyle et al., IBM Journal of Research and Development 49351 (2005). 